\title{
Predictive Value of Sympathetic Skin Response in Diagnosing Complex Regional Pain Syndrome: A Case-Control Study
}

\author{
Hyun Jung Kim, MD ${ }^{1,2}$, Hea Eun Yang, MD $^{3}$, Dae Hyun Kim, MD ${ }^{1,2}$, Yoon Ghil Park, MD, PhD ${ }^{1,2}$
}

${ }^{1}$ Department of Rehabilitation Medicine, Gangnam Severance Hospital, Seoul; ${ }^{2}$ Rehabilitation Institute of Neuromuscular Disease, Yonsei University College of Medicine, Seoul; ${ }^{3}$ Veteran Health Service Medical Center, Seoul, Korea

Objective To investigate the predictive value of the sympathetic skin response (SSR) in diagnosing complex regional pain syndrome (CRPS) by comparing three diagnostic modalities-SSR, three-phasic bone scans (TPBS), and thermography.

Methods Thirteen patients with severe limb pain were recruited. Among them, 6 were diagnosed with CRPS according to the proposed revised CRPS clinical diagnostic criteria described by the International Association for the Study of Pain. SSR was measured in either the hands or feet bilaterally and was considered abnormal when the latency was prolonged. A positive TPBS finding was defined as diffuse increased tracer uptake on the delayed image. Thermographic findings were considered positive if a temperature asymmetry greater than $1.00^{\circ} \mathrm{C}$ was detected between the extremities.

Results Five of 6 CRPS patients showed prolonged latency on SSR (83\% sensitivity). TPBS was positive in the 5 CRPS patients who underwent TPBS (100\% sensitivity). Thermography was positive in 4 of 5 CRPS patients who underwent the procedure ( $80 \%$ sensitivity). The remaining 7 non-CRPS patients differed on examination. SSR latencies within normal limit were noted in 4 of 7 non-CRPS patients ( $57 \%$ specificity). Results were negative in 4 of 5 non-CRPS patients who underwent TPBS ( $80 \%$ specificity), and negative in 3 of 5 non-CRPS patients who underwent thermography (60\% specificity).

Conclusion SSR may be helpful in detecting CRPS.

Keywords Complex regional pain syndromes, Galvanic skin response, Radionuclide imaging, Thermography

Received June 20, 2014; Accepted September 15, 2014

Corresponding author: Yoon Ghil Park

Department of Rehabilitation Medicine, Gangnam Severance Hospital, 211 Eonju-ro, Gangnam-gu, Seoul 135-720, Korea

Tel: +82-2-2019-3493, Fax: +82-2-3463-7585, E-mail: drtlc@yuhs.ac

(c) This is an open-access article distributed under the terms of the Creative Commons Attribution Non-Commercial License (http://creativecommons. org/licenses/by-nc/3.0) which permits unrestricted noncommercial use, distribution, and reproduction in any medium, provided the original work is properly cited.

Copyright $\odot 2015$ by Korean Academy of Rehabilitation Medicine

\section{INTRODUCTION}

Complex regional pain syndrome (CRPS) is a painful condition characterized by the presence of regional pain and sensory changes following a noxious event. The pain is accompanied by abnormal skin color, temperature change, abnormal sudomotor activity, or edema [1]. There are three stages of CRPS: stage 1, development of 
limb pain; stage 2, progression of symptoms and signs; stage 3, limitation of motion, shoulder-hand syndrome, contractures of the digits, waxy and trophic skin changes, and brittle, ridged nails. Clinical experience suggests that treatment is most effective when initiated during stage 1 immediately after establishing a diagnosis but before radiographic changes appear [2]. The early clinical presentation of CRPS may resemble other disorders, such as cervical nerve root impingement, Pancoast syndrome, vasculitis, migratory osteolysis, arteriovenous fistulae, progressive systemic sclerosis, disuse atrophy, and angioedema. CRPS is usually diagnosed on clinical examination, but diagnosis during early CRPS development can be difficult due to the lack of objective measures.

CRPS encompasses disorders characterized by spontaneous or stimulus-induced pain that is disproportionate to the inciting event and accompanied by a wide variety of autonomic and motor disturbances in varying combinations. Harden et al. [3] proposed new diagnostic criteria for CRPS.

Several studies evaluated the diagnostic modalities for CRPS, but none have compared SSR, TPBS, and thermography for CRPS diagnosis. Thus, the aim of this study was to compare the predictive value of sympathetic skin response (SSR), three-phasic bone scan (TPBS), and thermography in the diagnosis of CRPS.

\section{MATERIALS AND METHODS}

\section{Participants}

A total 13 patients with severe upper or lower limb pain evaluated at the Gangnam Severance Hospital from December 2011 to February 2013 were included. The patients were diagnosed using the proposed revised CRPS clinical diagnostic criteria [3], according to the International Association for the Study of Pain (IASP). The CRPS type was determined using the IASP diagnostic criteria for CRPS [4]. Six patients were diagnosed with CRPS, according to the revised CRPS clinical diagnostic criteria proposed by IASP [3]. Among them, 4 patients were diagnosed with CRPS-I and 2 patients with CRPS-II. Seven patients reported similar upper or lower limb pain, but they did not fulfill the proposed clinical diagnostic criteria for CRPS at the time of evaluation and until 8 months follow-up post-symptom onset.

\section{Sympathetic skin response}

Patients were informed on the SSR method prior to examination. Patients were positioned supine and relaxed on a secured bed in a silent examination room kept at $22^{\circ} \mathrm{C}-24^{\circ} \mathrm{C}$ or higher [5-10]. The skin temperature was maintained at $32^{\circ} \mathrm{C}-36^{\circ} \mathrm{C}$ [11-17]. The skin was cleansed and covered with electrolyte gel; active electrodes were then placed on the palm or sole and the reference electrode over the dorsum of the respective body part. The median nerve contralateral to the recorded site was stimulated and latency was measured. SSR was performed using EMG equipment (Medelec Synergy; Oxford Instruments Medical Ltd., Surrey, UK) that included a built-in monitor, bipolar stimulator, and discoid electrodes at the following settings: 10-30 mA stimulation intensity, 0.1 ms stimulation duration, $0.5-1,000 \mathrm{~Hz}$ frequency filter, $0.5 \mathrm{mV} / \mathrm{div}$ sensitivity, $0.5 \mathrm{sec} / \mathrm{div}$ sweep speed, 1 minute minimal interstimulus interval, irregular stimulus, and 3 [18]. The stimulus was delivered irregularly a 1-minute or greater interstimulus interval to avoid habituating the sympathetic skin response. Latency was measured at the initiation of the negative phase, and the shortest latency was chosen among three results. Knezevic and Bajada [11] previously reported that the mean palmar peripheral autonomic surface potential latency was $1.52 \pm 0.13 \mathrm{sec}$ onds and the mean plantar peripheral autonomic surface potential latency was $2.07 \pm 0.16$ seconds in 30 normal subjects. We considered the latency was prolonged if it was longer than $1 \mathrm{SD}$ ( $>1.65$ seconds in palmar and $>2.23$ seconds in plantar).

\section{Three-phasic bone scan}

TPBS was performed following an intravenous bolus of $20 \mathrm{mCi}$ of Tc-99m methylene diphosphonate. Sequential radionuclide blood flow (first phase) images were obtained, blood pool (second phase) images were obtained 2 to 3 minutes later, and delayed (third phase) images were obtained 3 to 4 hours later. Diffuse increased tracer uptake during the third phase, confirmed by a nuclear medicine physician, was considered a positive finding.

\section{Thermography}

Patients were informed of factors affecting the thermography result and were forbidden to receive any treatment or examination that could affect the thermography findings, such as physical activity, electromyography, 
Table 1. Patient characteristics and results

\begin{tabular}{cccllllc}
\hline Patient no. & Sex & Age & Diagnosis $^{\text {a) }}$ & SSR site & SSR (ms) & TPBS & Thermography $^{\text {b) }}$ \\
\hline 1 & M & 33 & CRPS type I & Sole & $1800 / 3510$ & CRPS & 5.28 \\
2 & M & 44 & CRPS type I & Palm & $1210 / 1620$ & CRPS & $>1$ \\
3 & M & 49 & CRPS type I & Sole & $3500 / 2230$ & CRPS & 3.86 \\
4 & M & 65 & CRPS type I & Palm & $1790 / 1830$ & CRPS & 0.64 \\
5 & M & 26 & CRPS type II & Sole & $1890 / 2900$ & CRPS & 4.11 \\
6 & F & 44 & CRPS type II & Palm & $1440 / 1940$ & NT & NT \\
7 & M & 51 & Contusion & Palm & $1780 / 1690$ & CRPS & 0.76 \\
8 & M & 68 & Arthritis & Palm & $1375 / 1375$ & Arthritis & NT \\
\hline 9 & M & 55 & Arthritis & Palm & $1500 / 1500$ & Arthritis & 5.53 \\
10 & F & 42 & Fibromyalgia & Sole & $2820 / 2550$ & NT & NT \\
11 & F & 68 & Arthritis & Palm & $1360 / 1820$ & NT & $<1$ \\
12 & F & 44 & Arthritis & Sole & $1660 / 1710$ & Arthritis & 2.37 \\
13 & F & 77 & Arthritis & Palm & $1461 / 1453$ & Arthritis & 0.78 \\
\hline
\end{tabular}

SSR, sympathetic skin response; TPBS, three-phasic bone scan; CRPS, complex regional pain syndrome; NT, not tested.

${ }^{a}$ According to the proposed revised CRPS clinical diagnostic criteria described by the International Association for the Study of Pain.

${ }^{b)}$ Temperature asymmetry between the affected and unaffected extremities $\left({ }^{\circ} \mathrm{C}\right)$.

alcohol for 24 hours previously, and tobacco for 2 hours previously. Before undergoing thermography, the patient was acclimated for 15 minutes unclothed in an isolated room maintained at a mean temperature of $23^{\circ} \mathrm{C} \pm 2^{\circ} \mathrm{C}$ and a relative humidity of $50 \%$. A temperature asymmetry between the affected and unaffected extremities greater than $1.00^{\circ} \mathrm{C}$ was considered a positive finding [19].

\section{Statistical analysis}

The sensitivity and specificity was calculated for SSR, TPBS, and thermography.

\section{RESULTS}

\section{Participants}

Subject demographics were shown in Table 1. Most of the patients were examined by SSR, TPBS, and thermography as part of the diagnostic confirmation. However, 1 CRPS patient and 1 non-CRPS patient underwent SSR alone; one non-CRPS patient did not undergo TPBS, and another non-CRPS patient did not undergo thermography.

\section{Sympathetic skin response}

The SSR findings in 5 of 6 CRPS patients indicated prolonged latency (83\% sensitivity). SSR latencies within
Table 2. Sensitivity and specificity

\begin{tabular}{lccc}
\hline & SSR & TPBS & Thermography \\
\hline Sensitivity (\%) & 83 & 100 & 80 \\
Specificity (\%) & 57 & 80 & 60 \\
\hline
\end{tabular}

SSR, sympathetic skin response; TPBS, three-phasic bone scan.

normal limit were noted in 4 of 7 non-CRPS patients ( $57 \%$ specificity) (Table 2).

\section{Three-phasic bone scan}

Positive findings were noted in all 5 CRPS patients who underwent TPBS (100\% sensitivity). The findings in the 5 non-CRPS patients who underwent TPBS were negative in 4 of 5 subjects ( $80 \%$ specificity) (Table 2 ).

\section{Thermography}

Thermography was positive in 4 of 5 CRPS patients $(80 \%$ sensitivity). In the 5 non-CRPS patients who underwent thermography, negative findings were noted in 3 of 5 subjects ( $60 \%$ specificity) (Table 2$)$.

\section{DISCUSSION}

This was the first comparative study of the diagnostic 
utility of SSR, TPBS, and thermography with the predictable value of SSR in CRPS diagnosis.

Patients with CRPS may experience sudomotor disturbances, such as hyperhidrosis or hypohidrosis [2,20-23]. The characteristic clinical signs and symptoms of autonomic dysfunction, including edema, skin temperature and color fluctuations, and hyperhidrosis, indicate a role of the sympathetic nervous system in the CRPS pathophysiology [24]. Sympathetic dysfunction is considered the main pathophysiological mechanism of CRPS [25-29].

The SSR is used to measure electrical activity changes occurring in the sweat glands following sympathetic stimulation $[5,30,31]$. Following an emotional or noxious stimulus, changes in sudomotor activity mediated by the sympathetic nervous system alters the skin's resistance to an electrical current.

There are few articles investigating the use of SSR for CRPS diagnosis.

Pankaj et al. [32] reported the diagnostic utility of SSR for diagnosing CRPS and compared SSR to TPBS. The study was conducted on 60 patients with CRPS affecting the hand; an absent response or a response with an peakto-peak amplitude $<50 \%$ compared to the contralateral hand was considered abnormal. Selcuk et al. [33] evaluated SSR in stroke patients with and without CRPS and healthy volunteers; and the onset latency, amplitude, and the number of phases was recorded. The SSR amplitude was significantly higher in patients with CRPS than in patients without it. Pankaj et al. [32] considered the reduction of amplitude of SSR as abnormal, while Selcuk et al. [33] considered the increment of amplitude of SSR as abnormal. Previous studies have shown conflicting results.

Mondelli et al. [34] found that the latency of SSR was prolonged in patients with primary Raynaud's phenomenon. Uncini et al. [6] reported that the SSR amplitude varied between people and that several factors influence the SSR amplitude. SSR latencies remained consistent even among significantly different recording sites, but not among different stimulation sites; therefore, measuring the SSR latency is important. We evaluated the latency of SSR for abnormality.

Several studies evaluated diagnostic modalities for CRPS. Park et al. [35] demonstrated that the sensitivity of CRPS-I diagnosis was $79.5 \%$ and $36.4 \%$ in thermography and TPBS, respectively. However, TPBS alone cannot pro- vide an accurate diagnosis; therefore, it is imperative that TPBS findings be integrated with clinical evaluation and other relevant findings.

According to Schurmann et al. [36], the sensitivity of plain radiography, TPBS, thermography, and MRI in diagnosing early post-traumatic CRPS-I was poor, but TPBS and MRI performed at 8 and 16 weeks later, and bilateral radiography at 8 weeks showed a high specificity (TPBS, $96 \%$ and $100 \%$; MRI, $78 \%$ and $98 \%$, respectively; radiography, 94\%). Moon et al. [37] reported that the diagnostic value of a positive TPBS result is low for CRPS, based on the Budapest research criteria. These results suggest that TPBS, thermography, MRI, and radiography cannot be used as screening tests, as they are unable to reliably differentiate between normal post-traumatic changes and those caused by CRPS-I. Clinical findings remain the diagnostic gold standard, while imaging serves as an auxiliary tool to establish diagnosis in suspected cases.

Pankaj et al. [32] found that TPBS is a very sensitive corroborative test to confirm a clinical suspicion of CRPS during the initial disease stages, but not in advanced cases. Alternatively, the SSR can detect sympathetic dysfunction in cases associated with a sweating abnormality and may have assistive diagnostic value in advanced cases of CRPS when TPBS is less reliable. Selcuk et al. [33] showed that SSR could be performed in stroke patients with CRPS even during early CRPS.

The present study was limited by small number of patients $(n=13)$. Further studies using a larger number of patients are necessary. Another limitation is that we added 1 standard deviation to the mean of the latency of SSR to judge the abnormality of SSR. The latency of SSR was shorter than $1650 \mathrm{~ms}$ in patient \#2; however, the latency of SSR of the affected side was longer than the unaffected side. If we consider the result of SSR as abnormal in patient \#2, the sensitivity of SSR is improved to $100 \%$.

The signs and symptoms of the patient are used in proposed clinical diagnostic criteria for CRPS, therefore it is highly subjective. Considering low cost, non-invasiveness and low possibility of complication from SSR, using SSR in diagnosing CRPS may be helpful.

In conclusion, SSR may be a helpful diagnostic test for CRPS. Although additional study is needed, SSR may serve as an objective tool to diagnose CRPS. 


\section{CONFLICT OF INTEREST}

No potential conflict of interest relevant to this article was reported.

\section{REFERENCES}

1. Stanton-Hicks M, Janig W, Hassenbusch S, Haddox JD, Boas R, Wilson P. Reflex sympathetic dystrophy: changing concepts and taxonomy. Pain 1995;63:12733.

2. Veldman PH, Reynen HM, Arntz IE, Goris RJ. Signs and symptoms of reflex sympathetic dystrophy: prospective study of 829 patients. Lancet 1993;342:1012-6.

3. Harden RN, Bruehl S, Stanton-Hicks M, Wilson PR. Proposed new diagnostic criteria for complex regional pain syndrome. Pain Med 2007;8:326-31.

4. Merskey H, Bogduk N. Classification of chronic pain: descriptions of chronic pain syndromes and definitions of pain terms. 2nd ed. Seattle: IASP Press; 1994.

5. Shahani BT, Halperin JJ, Boulu P, Cohen J. Sympathetic skin response: a method of assessing unmyelinated axon dysfunction in peripheral neuropathies. J Neurol Neurosurg Psychiatry 1984;47:536-42.

6. Uncini A, Pullman SL, Lovelace RE, Gambi D. The sympathetic skin response: normal values, elucidation of afferent components and application limits. J Neurol Sci 1988;87:299-306.

7. Lader MH, Montagu JD. The psycho-galvanic reflex: a pharmacological study of the peripheral mechanism. J Neurol Neurosurg Psychiatry 1962;25:126-33.

8. Levy DM, Reid G, Rowley DA, Abraham RR. Quantitative measures of sympathetic skin response in diabetes: relation to sudomotor and neurological function. J Neurol Neurosurg Psychiatry 1992;55:902-8.

9. Prout BJ. Independence of the galvanic skin reflex from the vasoconstrictor reflex in man. J Neurol Neurosurg Psychiatry 1967;30:319-24.

10. Wang SJ, Fuh JL, Shan DE, Liao KK, Lin KP, Tsai CP, et al. Sympathetic skin response and R-R interval variation in Parkinson's disease. Mov Disord 1993;8:151-7.

11. Knezevic W, Bajada S. Peripheral autonomic surface potential: a quantitative technique for recording sympathetic conduction in man. J Neurol Sci 1985;67:23951.

12. Dettmers C, Fatepour D, Faust H, Jerusalem F. Sym- pathetic skin response abnormalities in amyotrophic lateral sclerosis. Muscle Nerve 1993;16:930-4.

13. Gutrecht JA, Suarez GA, Denny BE. Sympathetic skin response in multiple sclerosis. J Neurol Sci 1993; 118:88-91.

14. Montagna P, Cortelli P, Avoni P, Marchello LP, Monari $\mathrm{L}$, Tinuper $\mathrm{P}$, et al. Abnormal sympathetic skin responses in thalamic lesions. Electroencephalogr Clin Neurophysiol 1992;85:225-7.

15. Niakan E, Harati Y. Sympathetic skin response in diabetic peripheral neuropathy. Muscle Nerve 1988; 11:261-4.

16. Solders G, Andersson T, Persson A. Central conduction and autonomic nervous function in HMSN I. Muscle Nerve 1991;14:1074-9.

17. Soliven B, Maselli R, Jaspan J, Green A, Graziano H, Petersen M, et al. Sympathetic skin response in diabetic neuropathy. Muscle Nerve 1987;10:711-6.

18. Vetrugno R, Liguori R, Cortelli P, Montagna P. Sympathetic skin response: basic mechanisms and clinical applications. Clin Auton Res 2003;13:256-70.

19. Niehof SP, Huygen FJ, van der Weerd RW, Westra M, Zijlstra FJ. Thermography imaging during static and controlled thermoregulation in complex regional pain syndrome type 1: diagnostic value and involvement of the central sympathetic system. Biomed Eng Online 2006;5:30.

20. Gierthmuhlen J, Maier C, Baron R, Tolle T, Treede RD, Birbaumer N, et al. Sensory signs in complex regional pain syndrome and peripheral nerve injury. Pain 2012;153:765-74.

21. Birklein F, Riedl B, Sieweke N, Weber M, Neundorfer B. Neurological findings in complex regional pain syndromes: analysis of 145 cases. Acta Neurol Scand 2000;101:262-9.

22. de Boer RD, Marinus J, van Hilten JJ, Huygen FJ, van Eijs F, van Kleef M, et al. Distribution of signs and symptoms of complex regional pain syndrome type I in patients meeting the diagnostic criteria of the International Association for the Study of Pain. Eur J Pain 2011;15:830.e1-8.

23. Sethna NF, Meier PM, Zurakowski D, Berde CB. Cutaneous sensory abnormalities in children and adolescents with complex regional pain syndromes. Pain 2007;131:153-61.

24. Borchers AT, Gershwin ME. Complex regional pain 
syndrome: a comprehensive and critical review. Autoimmun Rev 2014;13:242-65.

25. Bej MD, Schwartzman RJ. Abnormalities of cutaneous blood flow regulation in patients with reflex sympathetic dystrophy as measured by laser Doppler fluxmetry. Arch Neurol 1991;48:912-5.

26. Arner S. Intravenous phentolamine test: diagnostic and prognostic use in reflex sympathetic dystrophy. Pain 1991;46:17-22.

27. Chan RC, Chuang TY, Chiu FY. Sudomotor abnormalities in reflex sympathetic dystrophy. Zhonghua Yi Xue Za Zhi (Taipei) 2000;63:189-95.

28. Drucker WR, Hubay CA, Holden WD, Bukovnic JA. Pathogenesis of post-traumatic sympathetic dystrophy. Am J Surg 1959;97:454-65.

29. Marinus J, Moseley GL, Birklein F, Baron R, Maihofner C, Kingery WS, et al. Clinical features and pathophysiology of complex regional pain syndrome. Lancet Neurol 2011;10:637-48.

30. Shahani BT, Day TJ, Cros D, Khalil N, Kneebone CS. RR interval variation and the sympathetic skin response in the assessment of autonomic function in peripheral neuropathy. Arch Neurol 1990;47:659-64.

31. Gutrecht JA. Sympathetic skin response. J Clin Neurophysiol 1994;11:519-24.

32. Pankaj A, Kotwal PP, Mittal R, Deepak KK, Bal CS. Di- agnosis of post-traumatic complex regional pain syndrome of the hand: current role of sympathetic skin response and three-phase bone scintigraphy. J Orthop Surg (Hong Kong) 2006;14:284-90.

33. Selcuk B, Ersoz M, Inanir M, Kurtaran A, Akyuz M. Sympathetic skin responses in hemiplegic patients with and without complex regional pain syndrome. Neurol India 2006;54:279-82.

34. Mondelli M, de Stefano R, Rossi S, Aretini A, Romano C. Sympathetic skin response in primary Raynaud's phenomenon. Clin Auton Res 2009;19:355-62.

35. Park SH, Lee PB, Lim YH, Lee SY, Choi IY, Lee SJ, et al. The usefulness of three-phase bone scan and thermography for making the diagnosis of CRPS-I. Korean J Pain 2006;19:81-6.

36. Schurmann M, Zaspel J, Lohr P, Wizgall I, Tutic M, Manthey N, et al. Imaging in early posttraumatic complex regional pain syndrome: a comparison of diagnostic methods. Clin J Pain 2007;23:449-57.

37. Moon JY, Park SY, Kim YC, Lee SC, Nahm FS, Kim JH, et al. Analysis of patterns of three-phase bone scintigraphy for patients with complex regional pain syndrome diagnosed using the proposed research criteria (the 'Budapest Criteria'). Br J Anaesth 2012;108:65561. 